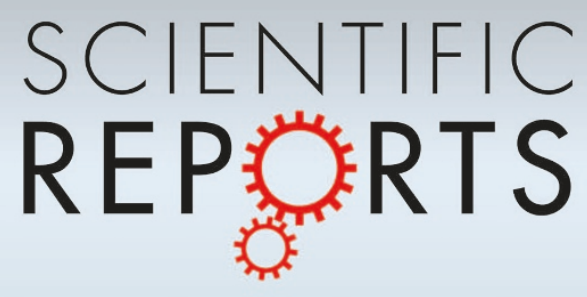

OPEN

SUBJECT AREAS:

NEUROGENETICS

MOLECULAR ENGINEERING

GENETIC MODELS

DEVELOPMENT

Received

2 May 2012

Accepted

15 June 2012

Published

6 July 2012

Correspondence and requests for materials should be addressed to

F.W. (fan.wang@ duke.edu)

\section{Intersectional Cre Driver Lines Generated Using Split-Intein Mediated Split-Cre Reconstitution}

\author{
Ping Wang' ${ }^{1}$ Tianrui Chen ${ }^{1,2}$, Katsuyasu Sakurai' ${ }^{1}$ Bao-Xia Han' ${ }^{1}$, Zhigang He ${ }^{3}$, Guoping Feng ${ }^{4}$ \\ \& Fan Wang'
}

'Department of Cell Biology, Box 3709, Duke University Medical Center, Durham, NC, ${ }^{2}$ Key Laboratory for Molecular Enzymology and Engineering of Ministry of Education, Jilin University, Changchun, People's Republic of China, ${ }^{3}$ Kirby Neurobiology Center, Children's Hospital Boston, Boston, MA, ${ }^{4}$ McGovern Institute for Brain Research, Department of Brain and Cognitive Sciences, MIT, Cambridge, MA.

Tissue and cell type highly specific Cre drivers are very rare due to the fact that most genes or promoters used to direct Cre expressions are generally expressed in more than one tissues and/or in multiple cell types. We developed a split-intein based split-Cre system for highly efficient Cre-reconstitution through protein splicing. This split-intein-split-Cre system can be used to intersect the expression patterns of two genes or promoters to restrict full-length Cre reconstitution in their overlapping domains. To test this system in vivo, we selected several conserved human enhancers to drive the expression of either Cre- $\mathrm{N}$-intein-N, or intein-C-Cre-C transgene in different brain regions. In all paired $\mathrm{CreN} / \mathrm{CreC}$ transgenic mice, Cre-dependent reporter was efficiently induced specifically in the intersectional expression domains of two enhancers. This split-intein based method is simpler to implement compared with other strategies for generating highly-restricted intersectional Cre drivers to study complex tissues such as the nervous system.

$\mathrm{T}$ he Cre-LoxP system is an enormously useful tool for deleting genes or inducing expression of genes in a tissue specific manner in mouse ${ }^{1}$. Most of the Cre drivers were generated using gene-based strategies taking advantage of restricted expression patterns of selected genes. These included inserting Cre cDNA into an endogenous gene locus, using promoters from known genes to drive Cre expression in transgenic mice ${ }^{2}$, or using BAC clones containing large fragments of genomic DNA flanking the desired gene to generate BAC transgenic lines $^{3}$. However, for a complex system such as the mammalian brain, genes that exhibit both sub-regional and neuronal-type specific expressions are very rare. Previously, a split-Cre $\alpha$-complementation system was developed in which the two fragments of the Cre recombinase (the Cre- $\mathrm{N}$ with the amino acids 19-59 and the Cre-C with residues 60-343) were found to spontaneously re-associate with each other to reconstitute the fulllength functional $\mathrm{Cre}^{4}$. The split-Cre system could be explored to generate highly restricted Cre driver lines using intersectional strategy, i.e. expressing the Cre-halves under the control of two different genes/promoters such that full-length Cre-reconstitution only occurs in their intersectional expression domains. To increase the efficiency of split-Cre $\alpha$-complementation, previously, a leucine-zipper based dimerization strategy was used ${ }^{5}$. However, efficient dimerization likely requires medium to high expression levels of each half-Cre to favor the equilibrium towards their association/interaction.

Here we explored the split-intein mediated protein splicing as an alternative means for efficient split-Cre reconstitution. An "intein" is a peptide within a precursor protein that is able to excise itself and at the same time rejoins the remaining portions of the protein (exteins) with a peptide bond, a process also called "proteinsplicing" ${ }^{6,7}$. Furthermore, a "split-intein" was found in the cyanobacterium Synechocystis sp. In this case, the catalytic subunit of DNA polymerase III is encoded by two separate genes, dnaE- $n$ and dnaE-c, which are located about $745 \mathrm{~kb}$ apart on the genome ${ }^{8,9}$. dnaE-n contains the N-terminal of the polymerase followed by a 124 -aa (amino acid) N-intein (IntN), whereas the dnaE-c product consists of a 36-aa C-intein (IntC) followed by the Cterminal sequences of the polymerase. The split-intein efficiently binds each other to "trans-splice" the two halves of dnaE peptides into one functional protein while excises itself out ${ }^{8,9}$. We reasoned that if the split-intein could trans-splice the $\mathrm{CreN}$ and $\mathrm{CreC}$ in mammalian cells, then because a peptide bond is formed, the two halves could no longer dissociate from each other, making this a very effective way of full Cre-reconstitution, and thus ideal for generating intersectional Cre drivers. 


\section{Results}

Generating split-intein tagged split-Cre constructs and testing Cre-reconstitution in culture. We constructed split-intein tagged split-Cre fusion genes: Cre-N-Intein-N and Intein-C-Cre-C (Figure 1). We first tested this split-intein-split-Cre system in cultured cortical slices. Control full length Cre or various split-Cre plasmids were co-electroporated with equal amount of GFP plasmids ex vivo into the isolated brain of the embryonic Rosa-loxp-STOPloxp-tdTomato mice ${ }^{10}$, followed by organotypic culture. As shown in Supplemental Figure S1, co-electroporation of the wildtype Cre plasmid led to the efficient excision of the STOP cassette and the expression of the tdTomato fluorescent reporter in transfected neurons. Each half of the split-intein-tagged split-Cre (Cre-N and Cre-C) alone did not have any recombinase activity, and thus no tdTomato expression (Supplemental Figure S1b-c). By contrast, when the Cre- $\mathrm{N}$ and $\mathrm{Cre}-\mathrm{C}$ were co-electroporated into the cortical slices, they reconstitute the Cre activity effectively, leading to tdTomato expression in majority of the GFP positive neurons. Thus, the split-intein can indeed mediate split-Cre reconstitution with high efficiency in mouse neuronal lineages.

Generating and characterizing transgenic lines expressing splitintein tagged split-Cre. To test this system in vivo, we decided to generate transgenic lines using different enhancers to drive the expression of Cre- $\mathrm{N}$ and $\mathrm{Cre}-\mathrm{C}$ in different but potentially overlapping domains. Comparative genomic studies have identified extremely conserved enhancer elements across human, rodents and other vertebrate species (http://enhancer.lbl.gov) ${ }^{11,12}$. Transient transgenesis assays in mouse embryos revealed that subsets of enhancers drive expression in various regions of the nervous system in embryonic mice (11,12, http://enhancer.lbl.gov/). From this enhancer database, we selected five enhancers for proof-ofprinciple experiments, with enhancer elements 752 and 611 driving Cre-N, and enhancers 1163, 23 and 218 driving Cre-C expression (Supplementary Figure S2b-c). To visualize the expression domains of individual split-Cre transgenes, we added IRES-LacZ after the Cre-N or Cre-C sequences (Figure 1a, Supplemental Figure S2a-b). We expect that full-length active Cre recombinase will only be reconstituted in the overlapping domains of the two enhancers in the Cre- $\mathrm{N}$ and Cre- $\mathrm{C}$ paired-transgenic mice (Figure 1b). To examine Cre reconstitution and Cre activity, we further crossed the paired-transgenic lines with a Cre-reporter line, Rosa26-loxp-STOP-loxp-PLAP ( ${ }^{13}$, abbreviated as Rosa ${ }^{\text {Plap/+}}{ }^{\text {}}$ ) (Figure 1c). PLAP stands for human placental alkaline phosphatase, and its activity can be detected using an AP-staining protocol (see Method). Here we briefly describe results from three pairs of Cre-N/Cre-C double transgenic lines. Suffice it to say, at embryonic day 11.5 (E11.5), all of the individual transgenic lines showed expression patterns similar to what had been reported for that enhancer element based on transient transgenesis assays ${ }^{12}$. In all three pairs of CreN/CreC double transgenic mice, we observed Cre-activity only in the intersectional domains of the split-Cre halves. To simplify the description, the enhancer-driven split-intein-tagged split-Cre-IRESLacZ transgenic lines will be simply referred to as $\mathrm{CreN}$ or $\mathrm{CreC}$, followed by the enhancer element (e.g. CreN752).

The CreN752/CreC1163 pair of double-transgenic line. Whole-mount X-gal staining at E11.5 showed that CreN752 and CreC1163 lines displayed different expression patterns of LacZ, and that the most obvious overlapping (intersectional) region is the trigeminal ganglion (TG) (arrows in Figure 2a). After sectioning, we found that CreC1163 was expressed in only a small number of scattered neurons in TG, as well as in epidermal tissues (Figure 2b, arrows in LacZ panels, and data not shown). By contrast, in CreN752 line, LacZ was present in many TG neurons (Figure $2 b, \mathrm{LacZ}$ panel), as well as in dorsal root ganglion (DRG) neurons (arrowheads in Figure 2a, and data not shown). When crossed to Rosa ${ }^{\text {Plap/+ }}$ reporter, neither CreN752 nor CreC1163 alone induced PLAP expression; whereas in triple transgenic CreN752; CreC1163; Rosa ${ }^{\text {Plap/+ }}$ mice, AP-staining nicely and selectively labeled scattered TG neurons and their axons (Figure 2b, Plap panel).

Since the TG is a clearly defined ganglion, we could perform quantitative counting of neuronal numbers to assess the efficiency of split-Cre reconstitution. Using two-color in situ hybridization, we found that $\mathrm{CreC}$ (driven by enhancer 1163) was expressed at low levels and in fewer cells compared with CreN (driven by enhancer

a

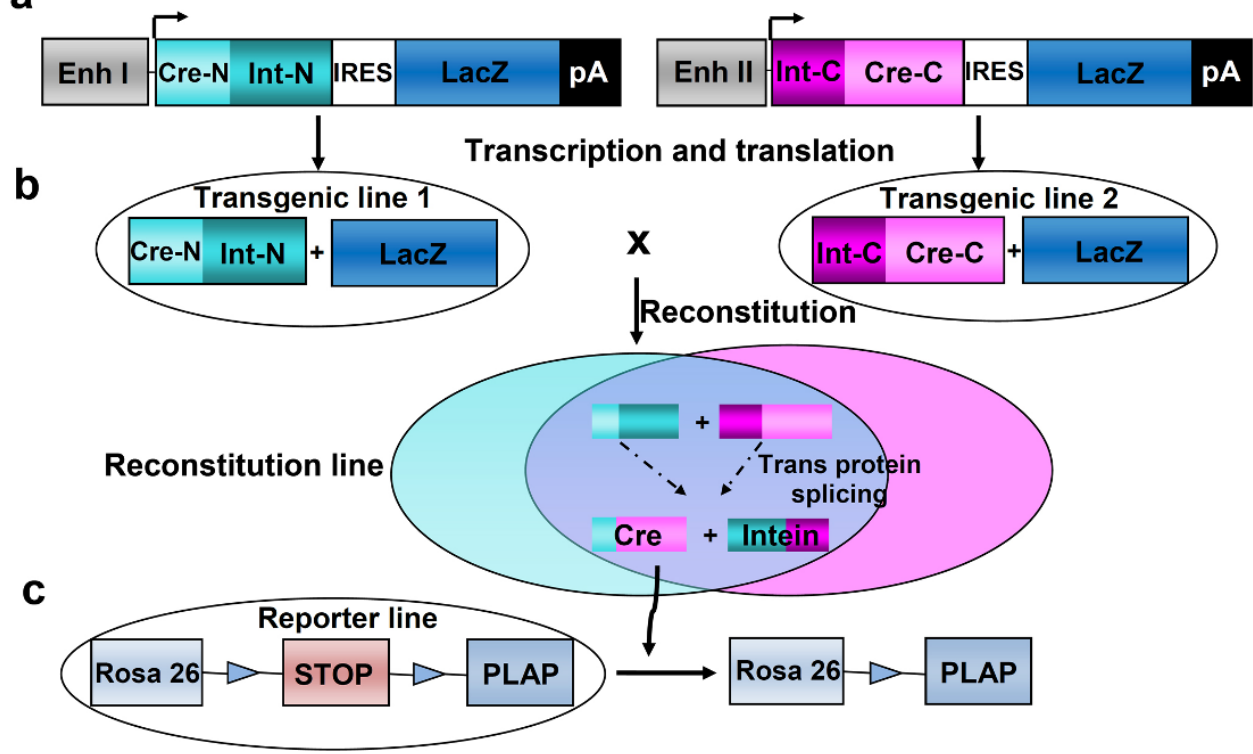

Figure 1 Schematic drawing of split-intein mediated split-Cre reconstitution system. (a) Schematic drawing of the structure of split-Cre transgenes: the expression of Cre-N-Intein-N or Intein-C-Cre-C followed by IRES-LacZ is driven by a selected enhancer. (b) IRES mediates co-expression of LacZ with the split-intein tagged split-Cre. After split-intein mediated protein trans-splicing, Cre- $\mathrm{N}$ and Cre-C is joint by a peptide bond to form the full Cre while the intein excise itself out. (c) Rosa26-loxp-STOP-loxp-PLAP (PLAP stands for human placenta alkaline phosphatase) reporter is used to visualize Cre reconstitution, we expect that STOP cassette is excised and PLAP is induced in the overlapping expression domain controlled by two enhancers. 
a

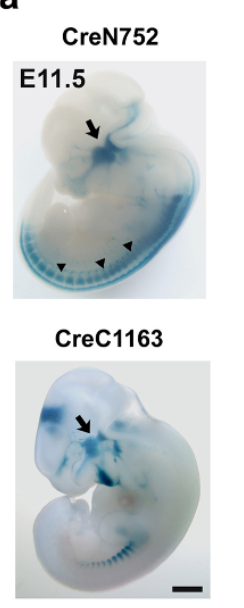

b

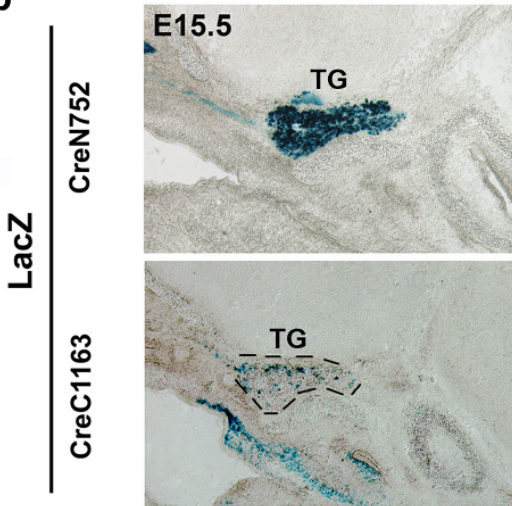

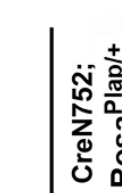

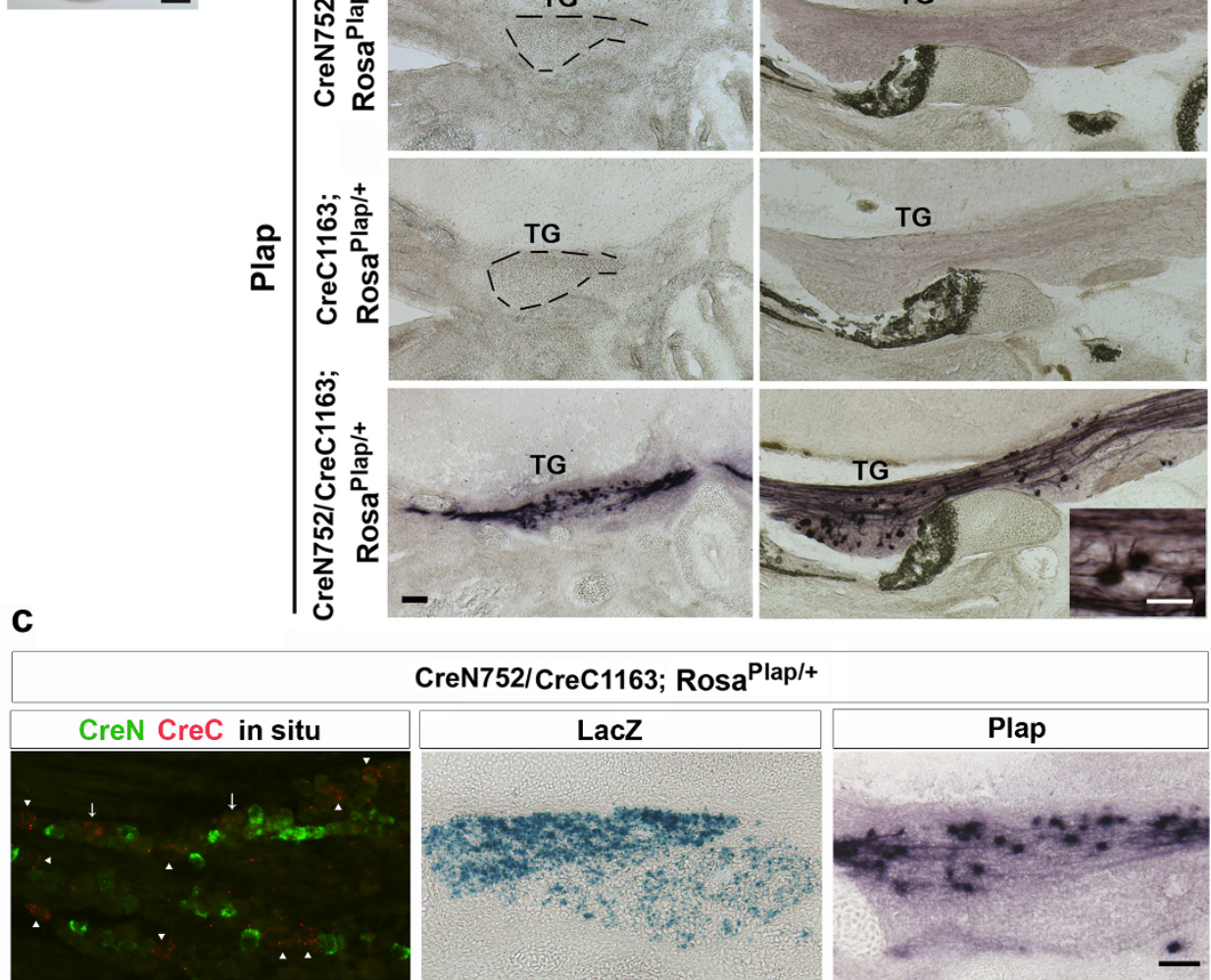

Figure $2 \mid$ Characterization of CreN752/CreC1163 paired double-transgenic mice. (a) Whole-mount LacZ staining of embryo of CreN752 and CreC1163 transgenic mice line at E11.5. LacZ were present in the trigeminal ganglion (TG) region of both lines (arrows). Note that in CreC1163, LacZ appeared to be expressed weaker than that in CreN752 and in part of TG. CreN752 but not CreC1163 is expressed in dorsal root ganglion (DRG) (arrowheads). Scale bar is $1 \mathrm{~mm}$. (b) Top two rows: Sagittal sections of trigeminal ganglion (TG) at E15.5 and P5 from CreN752 or CreC1163 transgenic mice stained with X-gal. Bottom three rows: sagittal sections of trigeminal ganglion (TG) from CreN752; Rosa ${ }^{\text {Plap }}{ }^{+/-}$, or CreC1163; Rosa ${ }^{\text {Plap+l- }}$, or CreN752/CreC1163; Rosa ${ }^{\text {Plap+l- }}$ mice stained for PLAP activity. Scale bar is $100 \mu \mathrm{m}$. (c) Left panel: CreN752 (green) and CreC1163 (red) two-color in situ showing most of the Crec1163 positive cells (arrowheads) lacking CreN752 expression, and the putative CreN/CreC double-positive neurons (arrows) showing low level CreN expression. Right two panels: the rare co-expression of CreN/CreC resulted in the comparable level of sparse PLAP-positive neurons in the trigeminal ganglion. Scale bar is $100 \mu \mathrm{m}$.

752) (Figure 2c), consistent with the $\mathrm{X}$-gal/LacZ staining results (Figure 2b). Importantly, CreN752 and CreC1163 were expressed by largely non-overlapping populations of neurons in the TG (Figure 2c). In fact, the rare CreN/CreC double-positive all showed low level of CreC (arrows in Figure 2c). On average, only $4.2 \pm 0.8 \%$ of the total number of $\mathrm{CreN}$ and/or $\mathrm{CreC}$ single positive neurons were $\mathrm{CreN} / \mathrm{CreC}$ double-positive. Interestingly, the number of PLAPstained neurons was $6.7 \pm 1.4 \%$ of the total number of $\mathrm{X}$-gal/LacZpositive TG neurons (Figure 2c), suggesting all neurons that had coexpression of CreN752 and CreC1163 reconstituted Cre-activity and induced reporter expression. It is likely that some neurons with weak
CreN and CreC co-expression, below the in situ detection threshold, also had full Cre-activity. These results strongly suggest that splitintein mediated Cre-reconstitution is highly efficient.

The CreN611/CreC23 pair of double-transgenic line. At E11.5, the intersectional expression domain of enhancer element 611 and 23 appeared to be in the pre-rhombomere and rhombomere regions, which later would develop into part of the cerebellum and hindbrain (arrow in Figure 3a). Sectioning through the brainstems of postnatal day 3 (P3) mice revealed that in both CreN611 and CreC23 line, LacZ was expressed in small number of neurons in the reticular regions of 
a

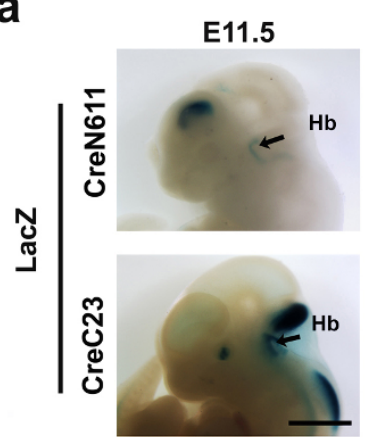

b

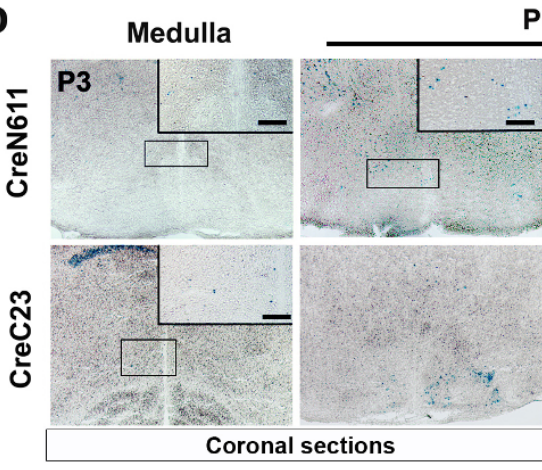

Pons

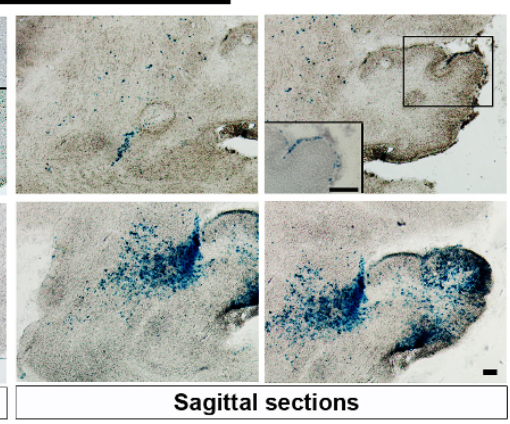

C |

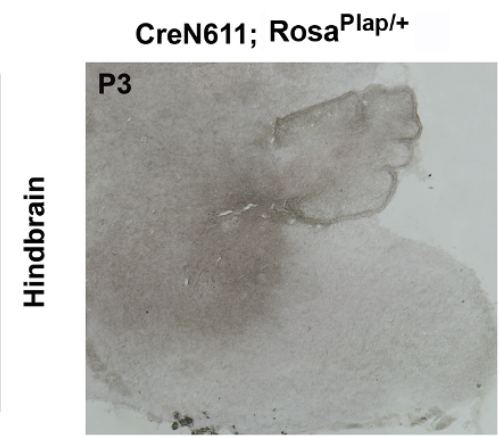

CreC23; Rosa $a^{\text {Plap/+ }}$

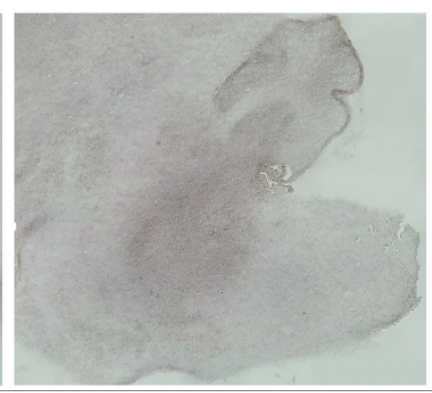

Sagittal sections
CreN611/CreC23; RosaPlap/+

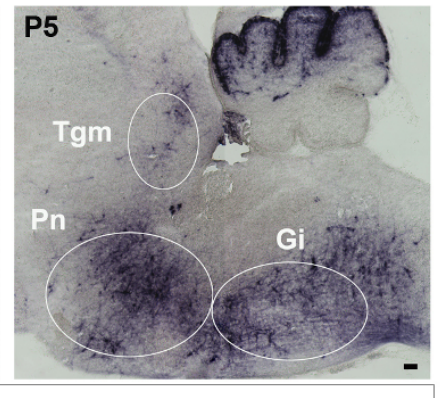

d

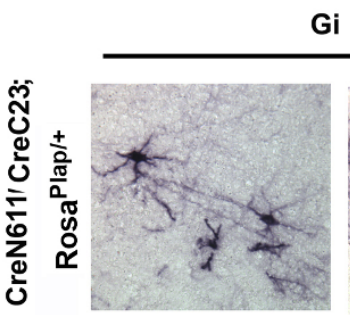

Gi

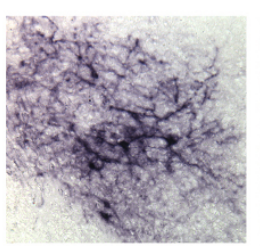

Pn

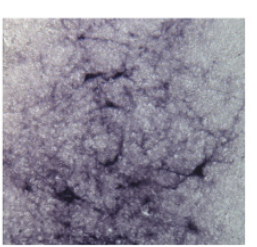

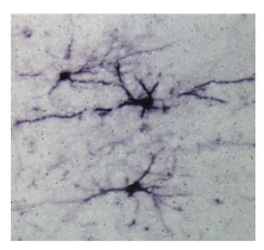

Figure 3 Characterization of CreN611/CreC23 paired double transgenic mice. (a) Whole-mount LacZ staining of embryo of CreN611 and CreC23 transgenic mice lines at E11.5, showing the overlapping region of expression of LacZ in the hindbrain region (arrows). (b) Representative coronal or sagittal sections from P3 brains of CreN611 or CreC23 lines. Both lines showed LacZ expression in scattered cells in medulla and pons. CreC23 is strongly expressed in cerebellum, while CreN611 drives lacZ expression in cells at the border of dorsal cerebellum (see inset). (c) Representative sagittal sections

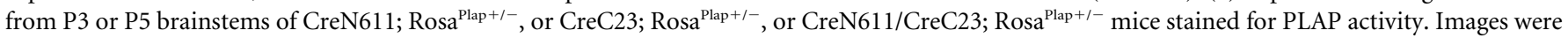
stitched together manually and the final composite images of brainstems were shown. Representative images from circled regions are shown in d. (d) Representative high magnification coronal images showing PLAP expression in the brainstem of CreN611/CreC23; Rosa ${ }^{\text {Plap }+1-} \mathrm{mice}$. Gi, Gigantocellular reticular nucleus; Pn, Pontine reticular nucleus; Tgm, Tegmental nucleus. Scale bars: $1 \mathrm{~mm}(\mathrm{a}), 100 \mu \mathrm{m}$ (b-d).

the medulla and the pons (Figure $3 \mathrm{~b}$ ). In addition, CreC23 showed strong lacZ expression within the cerebellum and in subregions of the tegmental area (Figure 3b). CreN611 on the other hand, had overall low level of LacZ expression in the superficial layer of the dorsal cerebellum (inset in Figure $3 b$, cerebellum panel), as well as in scattered cells in tegmentum. Again, neither CreN611, nor CreC23 alone led to PLAP expression when crossed to Rosa ${ }^{\text {Plap/+ }}$ reporter. Importantly, in triple transgenic CreN611; CreC23; Rosa ${ }^{\text {Plap } /+}$ mice, PLAP expression was induced and restricted to the brainstem and cerebellum: in subsets of neurons in the gigantocellular (Gi) and pontine reticular formation (Pn), in the tegmental nuclei (Tgm), and in the outer layers of the dorsal cerebellum (Figure $3 \mathrm{c}-\mathrm{d}$ ). Thus, even though CreN611 was expressed at relatively low levels in the brainstem, PLAP was induced in the intersectional domains of the two lines, again suggesting that split-intein-mediated Crereconstitution was very efficient.

The CreN611/CreC218 pair of transgenic line. A CreN-line should be able to pair with any CreC-lines to drive unique intersectional expressions. To examine this, we next paired CreN611 with a different CreC-line: CreC218. At E11.5, whole-mount X-gal/LacZ staining showed that both enhancers drive expression in forebrain and hindbrain, but the patterns are different (Figure $4 \mathrm{a}$, left panels). After sectioning of the E11.5 brain, we found that enhancer CreN611 is not expressed in the neocortex at this stage, but in the region near the ventral part of the developing hippocampus and in choroid plexus epithelium (Figure 4a, CreN611 sagittal section). By contrast, CreC218 is expressed in the developing neocortex (Figure 4a, CreC218 sagittal section). Thus, at E11.5, the CreN611 and Cre$\mathrm{C} 218$ were in non-overlapping domains in the forebrain. At P3, sections of the brains showed that both enhancers still drive expressions in largely distinct layers in the cortex (in medial-posterior regions), and in subsets of cells of the superior colliculus and brainstem (Figure $4 \mathrm{~b}$ ). All three mentioned regions potentially contain small number of cells co-expressing CreN611 and CreC218. Indeed in triple transgenic CreN611; CreC218; Rosa $^{\text {Plap/+ }}$ mice, reconstituted Cre-induced PLAP expression in these predicted intersectional domains, including subsets of neurons in the brainstem reticular 
a

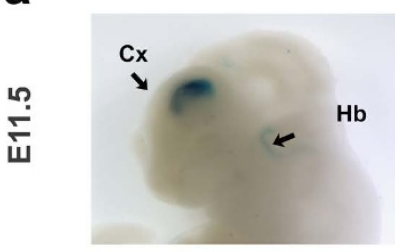

CreC218

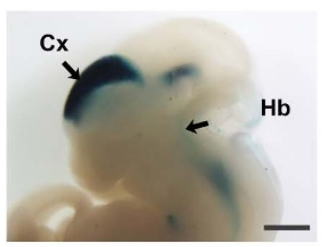

CreN611

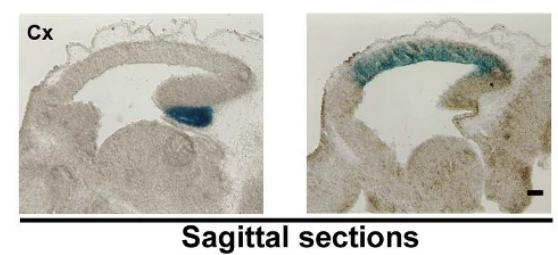

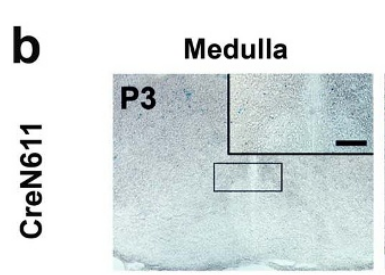

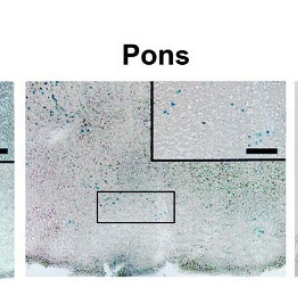

Superior Colliculus
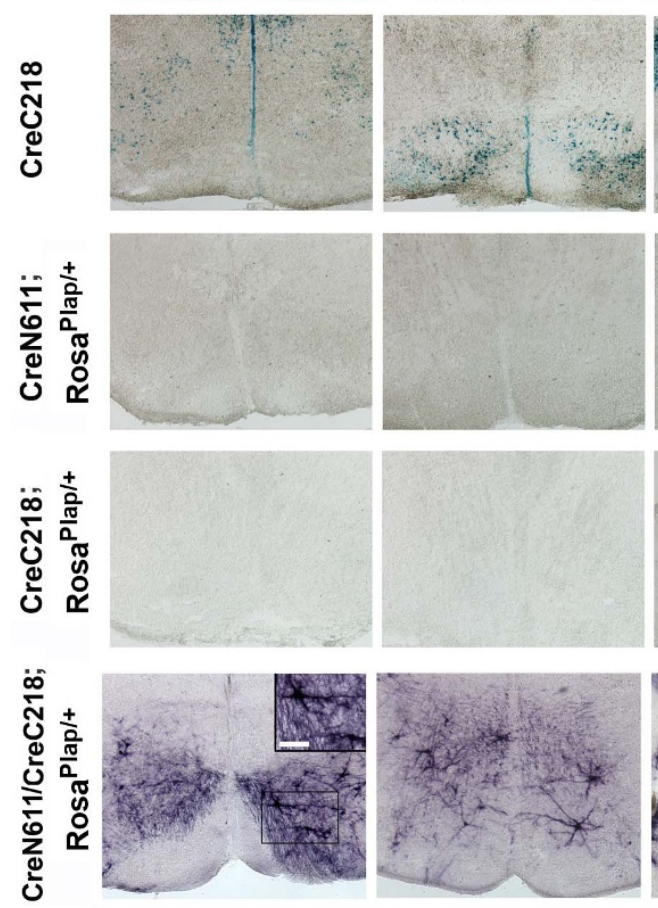

Retrosplenial Dysgranular Cortex

Cortex
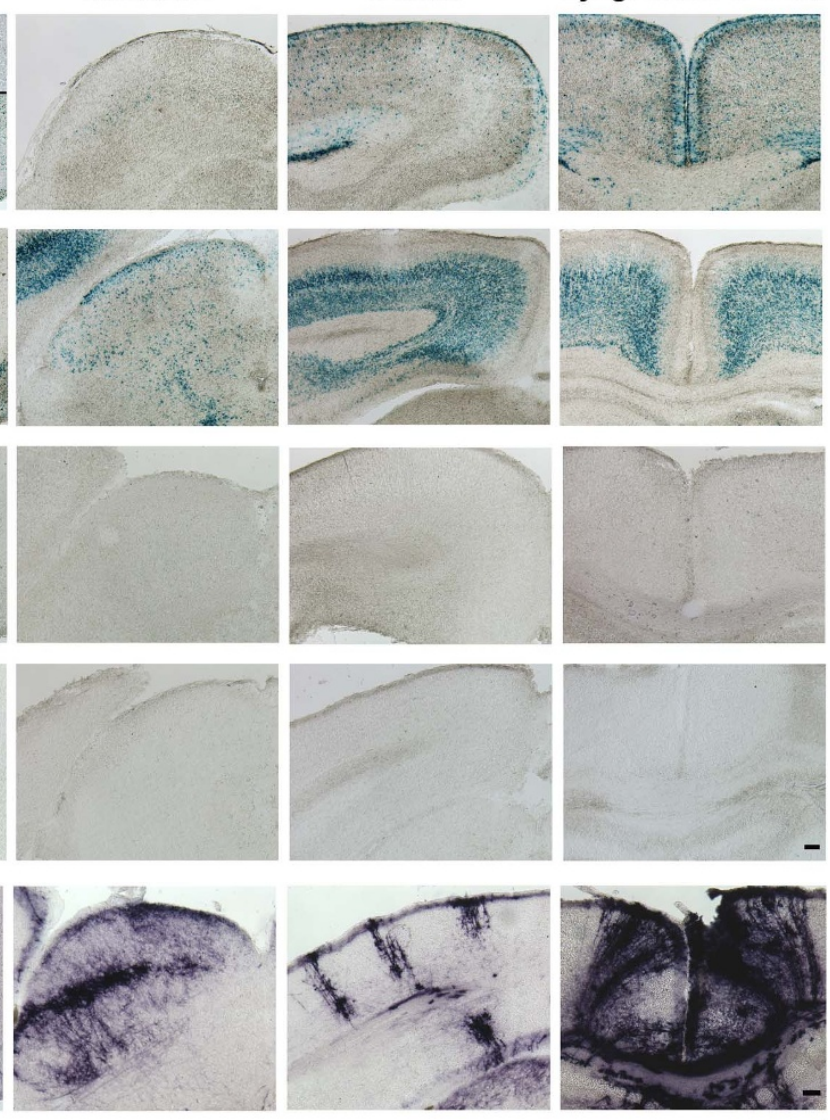

\section{Coronal sections}

Figure 4 | Characterization of CreN611/CreC218 paired double-transgenic mice. (a) Whole-mount LacZ staining of embryos from CreN611 and CreC218 transgenic mice lines at E11.5 are shown on the left, and sagittal sections of the E11.5 brains from these two lines are shown on the right. Arrows point to cortex $(\mathrm{Cx})$ and hindbrain $(\mathrm{Hb})$. Note that sectioning of the brain showed that the two lines have non-overlapping expression patterns in the neocortex at this stage. Scale bar is $1 \mathrm{~mm}$. (b) Representative coronal sections from P3 brains of CreN611 or CreC218 stained with X-gal; and representative coronal sections from P3 brains of CreN611; Rosa ${ }^{\text {Plap }^{+/-}}$, or CreC23; Rosa ${ }^{\text {Plap }+/-}$, or CreN611; CreC218; Rosa ${ }^{\text {Plap }+/-}$ mice stained for PLAP activity. Scale bar is $100 \mu \mathrm{m}$.

formation (in pons and medulla), the middle layer of the superior colliculus, and a few columns of neurons in the cortex (in the visual and the retrosplenial dysgranular cortex) (Figure $4 \mathrm{~b}$ ). The scattered cortical columns probably resulted from rare co-expressions of CreN/CreC in some progenitor cells, and after Cre-reconstitution, PLAP was expressed in all of the progenies of the progenitors.

\section{Discussion}

Our study shows that split-intein mediated split-Cre reconstitution through protein splicing is a very valuable and versatile tool for exploiting intersectional strategies to generate highly specific Cre drivers. There are more and more "floxed" alleles of mouse genes being generated $^{14}$, yet cell-type restricted Cre drivers are severely lacking. Using two recombinases, Flip and Cre, in one mouse is one way to intersect two expression domains, and this strategy has been successfully used in mapping cell fate and lineages ${ }^{15,16}$. In this approach, one gene/promoter is used to drive the expression of Flip recombinase, and the other gene/ promoter directs the expression of frt-STOP-frt cassette followed by the Cre gene, such that Cre is expressed after Flip-mediated excision of the frt-STOP-frt cassette. However, this Flip/Cre approach has not been employed to conditionally delete genes of interest in the desired intersectional domains, as this method involve the use and retention of multiple recombination sites. For example, to generate a knock-in allele of Flip, one normally uses loxP-neo-loxP cassette as a removable selection marker. The Cre knock-in or transgenic allele requires a frt-neofrt cassette placed in front of Cre for Flip-dependent expression. Furthermore, most of the "floxed" alleles were also generated with a frt-neo-frt selection marker ${ }^{14}$. Thus, when the Flip, Cre, and floxed alleles are crossed into one mouse, the multiple recombination sites coupled with two recombinases for multiple recombination events could cause potential complications such as chromosome crossovers and genomic instability in the triple transgenic mice. 
We believe that our split-intein-split-Cre system offers a simpler alternative for expressing full Cre in restricted domains without invoking multiple recombination events or worrying of the multiple loxP and frt sites, and is compatible with most of the floxed alleles that normally retain frt sites. Furthermore, the half-Cre lines (CreN or CreC lines) can be used in different combinations, thereby a small number of $\mathrm{CreN}$ and $\mathrm{CreC}$ lines can result in a large number of combinatorial pairs to drive expression in different cell types. For example, CreN expressed under the control of a "master" gene/marker of a tissue or a brain region, can be paired with different CreC lines controlled by other genes expressed in selected subtype of cells within that tissue or brain region, even though those subtypemolecular makers themselves may be expressed in other tissues, together, one could easily generate many tissue/region and cell-type specific Cre drivers.

\section{Methods}

Construction of Cre-N-Intein-N and Intein-C-Cre-C fusion proteins. To generate the Cre-N-Intein-N and Intein-C-Cre-C fusion proteins, the N- (aa 19-59) and Cterminal (aa 60-343) fragment of Cre recombinase, were first PCR amplified. The N terminal fragment $(\mathrm{Cre}-\mathrm{N})$ is fused to the intein- $\mathrm{N}$ which contains $123 \mathrm{~N}$-terminal amino acids of the intein sequence of DnaE split intein to yield Cre-N-Intein-N. In a similar manner, the $\mathrm{C}$ terminal fragment $(\mathrm{Cre}-\mathrm{C})$ is linked to the intein- $\mathrm{C}$ which contains 36 -aa intein sequence, in the order of Intein-C-Cre-C. For testing the splitintein tagged split-Cre in cultured cortical slices, the Cre-N-Intein-N or Intein-CCre-C fragment was sub-cloned into pCAGGS expression vector (Addgene).

DNA sequences of the split-intein-tagged split-Cre. Cre-N-Intein-N:

ATGACGAGTGATGAGGTTCGCAAGAACCTGATGGACATGTTCAGGGATCGCCAGGCGTTTTCTGAGCATACCTGGAAAATGCTTCTGTCCGTTTGCCGGTCGTGGGCGGCATGGTGCAAGTTGAATAAATTTGCGGAATATTGCCTCAGTTTTGGCACCGAAATTTTAACCGTTGAGTACGGCCCATTGCCCATTGGCAAAATTGTGAGTGAAGAAATTAATTGTTCTGTGTACAGTGTTGATCCAGAAGGGAGAGTTTACACCCAGGCGATCGCCCAATGGCATGACCGGGGAGAGCAGGAAGTATTGGAATATGAATTGGAAGATGGTTCAGTAATCCGAGCTACCTCTGACCACCGCTTTTTAACCACCGATTATCAACTGTTGGCGATCGAAGAAATTTTTGCTAGGCAACTGGACTTGTTGACTTTAGAAAA TATTAAGCAAACTGAAGAAGCTCTTGACAACCATCGTCTTCCCTTTCCATTACTTGACGCTGGGACAATTAAATAA

Intein-C-Cre-C

ATGGTTAAAGTTATCGGTCGTCGTTCCCTCGGAGTGCAAAGAATATTTGATATTGGTCTTCCCCAAGACCATAATTTTCTGCTAGCCAATGGGGCGATCGCCGCCAATTGTTTTAACAAATCCAACCGGAAATGGTTTCCCGCAGAACCTGAAGATGTTCGCGATTATCTTCTATATCTTCAGGCGCGCGGTCTGGCAGTAAAAACTATCCAGCAACATTTGGGCCAGCTAAACATGCTTCATCGTCGGTCCGGGCTGCCACGACCAAGTGACAGCAATGCTGTTTCACTGGT TATGCGGCGGATCCGAAAAGAAAACGTTGATGCCGGTGAACGTGCAAAACAGGCTCTAGCGTTCGAACGCACTGATTTCGACCAGGTTCGTTCACTCATGGAAAATAGCGATCGCTGCCAGGATATACGTAATCTGGCATTTCTGGGGATTGCTTATAACACCCTGTTACGTATAGCCGAAATTGCCAGGATCAGGGTTAAAGATATCTCACGTACTGACGGTGGGAGAATGTTAATCCATATTGGCAGAACGAAAACGCTGGTTAGCACCGCAGGTGTAGAGAAGGCACTTAGCCTGGGGGTAACTAAACTGGTCGAGCGATGGATTTCCGTCTCTGGTGTAGCTGATGATCCGAATAACTACCTGTTTTGCCGGGTCAGAAAAAATGGTGTTGCCGCGCCATCTGCCACCAGCCAGCTATCAACTCGCGCCCTGGAA GGGATTTTTGAAGCAACTCATCGATTGATTTACGGCGCTAAGGATGACTCTGGTCAGAGATACCTGGCCTGGTCTGGACACAGTGCCCGTGTCGGAGCCGCGCGAGATATGGCCCGCGCTGGAGTTTCAATACCGGAGATCATGCAAGCTGGTGGCTGGACCAATGTAAATATTGTCATGAACTATATCCGTAACC TGGATAGTGAAACAGGGGCAATGGTGCGCCTGCTGGAAGATGGCGATTAG

Generation of split-Cre transgenic mice. The Cre-N-Intein-N or Intein-C-Cre-C fragment was inserted into the unique NcoI site of the Hsp68LacZ gateway vector containing the human enhancers of interest, and a small promoter of the heat-shock protein 68 as well as the lacZ reporter gene, respectively ${ }^{11}$. Linearized transgenes were submitted to the Duke University transgenic facility for pronuclear injection to obtain transgenic founders. All animal procedures were performed according to protocols approved by The Duke University Institutional Animal Care and Use Committee. $\mathrm{X}$-Gal staining, AP staining, and in situ hybridization were performed following previously described protocols $s^{12,13,17}$.

For whole-mount X-gal staining, embryos were dissected out from timed-pregnant female and fixed in $4 \%$ PFA/PBS on ice for 30 mins, rinsed with buffer A (100 mM phosphate buffer, pH 7.4, $2 \mathrm{mM} \mathrm{MgCl} 2$ and $5 \mathrm{mM}$ EGTA) twice, for $5 \mathrm{~min}$ and $30 \mathrm{~min}$ each at room temperature, and followed by two washes of $5 \mathrm{~min}$ at room temperature with buffer B (100 mM phosphate buffer, $\mathrm{pH} 7.4,2 \mathrm{mM} \mathrm{MgCl} 2,0.01 \%$ sodium desoxycholate, and $0.02 \%$ Nonidet P40). Embryos were stained for blue color by exposure in the dark at $37 \mathrm{C}$ in buffer $\mathrm{C}$ (buffer B, plus $5 \mathrm{mM}$ potassium-ferricya- nide, $5 \mathrm{mM}$ potassium-ferrocyanide, and $1 \mathrm{mg} / \mathrm{ml}$ of X-Gal). For sections, dissected embryos were post-fixed in 4\% PFA/PBS on ice for 1 hour, and saturated in $30 \%$ sucrose/PBS at $4{ }^{\circ} \mathrm{C}$ overnight. Sections were collected with a cryostat at $20-25 \mu \mathrm{m}$ thickness, and staining was conducted same as described for the whole-mount staining.

For AP staining to detect PLAP activity, sections were collected using a cryostat at $20 \sim 40 \mu \mathrm{m}$ thickness. The sections were post-fixed with $4 \%$ PFA/PBS at room temperature for 1 hour, followed by inactivation step at $65^{\circ} \mathrm{C}$ for 6 hours in PBS. For the detection of AP activity, the sections were rinsed with wash buffer (0.1 M Tris- $\mathrm{HCl}$, $\mathrm{pH} 7.5,0.1 \mathrm{M} \mathrm{NaCl})$ and then developed in staining solution $[1: 50$ NBT/BCIP stock solution (Roche), 0.1 M Tris-HCl, pH 9.5, 0.1 M NaCl, $5 \mathrm{mM} \mathrm{MgCl} 2]$.

For fluorescent two-color in situ hybridization, first the cDNA sequences for the $\mathrm{CreN}$ and $\mathrm{CreC}$ fragment were used as template for in vitro transcription to generate either digoxigenin-UTP (Roche) or FITC-UTP (Roche) labeled antisense probes for $\mathrm{CreN}$ or $\mathrm{CreC}$, respectively. The two-color in situ hybridization was performed as described previously ${ }^{17}$.

Cell counting. Multiple E15.5 embryos from CreN752; CreC1163, Rosa ${ }^{\text {Plap } /+}$ were used for two-color in situ hybridization experiments, X-gal staining, or AP staining. Trigeminal ganglia were serial sectioned onto alternating slides for X-gal and AP staining, so adjacent section pairs can be compared. 15 randomly selected sections or alternating section pairs from each type of experiments were used for counting the number of $\mathrm{CreN}+, \mathrm{CreC}+$, or $\mathrm{CreN}+\mathrm{CreC}+$ (double positive), as well as $\mathrm{LacZ}+$, or $\mathrm{Plap}+$ neurons. The average percentage of $\mathrm{CreN}+\mathrm{CreC}+$ double positive neurons out of the total $\mathrm{CreN}+$ and/or $\mathrm{CreC}+$ neurons was calculated. Similarly, the average percentage of Plap + neurons over the total number of LacZ + neurons was calculated.

1. Nagy, A. Cre recombinase: the universal reagent for genome tailoring. Genesis. 26, 99-109 (2000).

2. Nagy, A., Mar, L. \& Watts, G. Creation and use of a cre recombinase transgenic database. Methods Mol Biol. 530, 365-78 (2009).

3. Gong, S. et al. A gene expression atlas of the central nervous system based on bacterial artificial chromosomes. Nature. 425, 917-25 (2003).

4. Casanova, E. et al. Alpha complementation in the Cre recombinase enzyme. Genesis. 37, 25-9 (2003).

5. Hirrlinger, J. et al. Split-cre complementation indicates coincident activity of different genes in vivo. PLoS One. 4, e4286 (2009).

6. Liu, X. Q. Protein-splicing intein: Genetic mobility, origin, and evolution. Annu Rev Genet. 34, 61-76 (2000).

7. Xu, M. Q. \& Evans, T. C. Jr. Recent advances in protein splicing: manipulating proteins in vitro and in vivo. Curr Opin Biotechnol. 16, 440-6 (2005).

8. Evans, T. C., Jr. et al. Protein trans-splicing and cyclization by a naturally split intein from the dnaE gene of Synechocystis species PCC6803. J Biol Chem. 275, 9091-4 (2000).

9. Wu, H., Hu, Z. \& Liu, X. Q. Protein trans-splicing by a split intein encoded in a split DnaE gene of Synechocystis sp. PCC6803. Proc Natl Acad Sci U S A. 95, 9226-31 (1998).

10. Arenkiel, B. R. et al. Activity-induced remodeling of olfactory bulb microcircuits revealed by monosynaptic tracing. PLoS One. 6, e29423 (2011).

11. Pennacchio, L. A. et al. In vivo enhancer analysis of human conserved non-coding sequences. Nature. 444, 499-502 (2006).

12. Visel, A. et al. Ultraconservation identifies a small subset of extremely constrained developmental enhancers. Nat Genet. 40, 158-60 (2008).

13. da Silva, S. et al. Proper formation of whisker barrelettes requires peripheryderived Smad4-dependent TGF-beta signaling. Proc Natl Acad Sci U S A. 108, 3395-400 (2011).

14. Skarnes, W. C. et al. A conditional knockout resource for the genome-wide study of mouse gene function. Nature. 474, 337-42 (2011).

15. Dymecki, S. M. \& Kim, J. C. Molecular neuroanatomy's "Three Gs": a primer. Neuron. 54, 17-34 (2007).

16. Dymecki, S. M., Ray, R. S. \& Kim, J. C. Mapping cell fate and function using recombinase-based intersectional strategies. Methods Enzymol. 477, 183-213 (2010).

17. Hasegawa, H. \& Wang, F. Visualizing mechanosensory endings of TrkCexpressing neurons in HS3ST-2-hPLAP mice. J Comp Neurol. 511, 543-56 (2008).

\section{Acknowledgement}

We thank Dr. Paul Liu at Dalhousie University, Canada for the split-intein plasmids. We thank Dr. Pennacchio at Lawrence Berkeley National Laboratory for all the human enhancer elements. This work is supported by a grant from NIH RC1NS068612 (Z.H., subcontract F.W), DA028302 (to F.W); and G.F is supported by R01 MH081201 and Simons Foundation Autism Research Initiative.

\section{Author contributions}

$\mathrm{KS}, \mathrm{ZH}, \mathrm{FG}, \mathrm{FW}$ designed the experiments and constructs; PW, TC and KS performed experiments; PW performed data analyses with the help of TC; PW and FW wrote the manuscript with inputs from $\mathrm{ZH}$ and FG. 


\section{Additional information}

Supplementary information accompanies this paper at http://www.nature.com/ scientificreports

Competing financial interests: The authors declare no competing financial interests.
License: This work is licensed under a Creative Commons

Attribution-NonCommercial-ShareAlike 3.0 Unported License. To view a copy of this license, visit http://creativecommons.org/licenses/by-nc-sa/3.0/

How to cite this article: Wang, P. et al. Intersectional Cre Driver Lines Generated Using Split-Intein Mediated Split-Cre Reconstitution. Sci. Rep. 2, 497; DOI:10.1038/srep00497 (2012). 